Table Agedistribution of the Chlamydia trachomatis positive pregnant women

\begin{tabular}{lll}
\hline & \multicolumn{2}{l}{ Number } \\
\cline { 2 - 3 } $\begin{array}{ll}\text { Age } \\
\text { (in years) }\end{array}$ & Total & $\begin{array}{l}\text { Chlamydia } \\
\text { positive }\end{array}$ \\
\hline$\leqslant 20$ & 28 & 3 \\
$21-25$ & 32 & 2 \\
$26-30$ & 10 & 1 \\
$31-35$ & 9 & 0 \\
$36-40$ & 6 & 2 \\
$\geqslant 41$ & 3 & 0 \\
Unknown & 13 & 1 \\
\hline
\end{tabular}

Kallestad diagnostics, Texas USA), with an epifluorescence microscope (Zeiss) at a magnification of $\times 400$. A specimen was considered as positive when ten or more apple-green fluorescent-stained elementary bodies were present.

The mean age of the women who participated in the study was 23.8 years (the age of 13 women was unknown).

The prevalence of Chlamydia trachomatis in the pregnant women examined was 9\% (9 out of 101 women). Remarkably, the highest prevalence 2 out of $6(33 \%)$ was found in the age group 36-40 years (table).

Although the prevalence percentages in the present investigation were of the same order as those found in other African countries ${ }^{3-5}$ one has to take into account that different methods were used in the studies described. Therefore it is rather difficult to compare the data from the different surveys.

In view of the relatively small number of neonates $(n=59)$ tested, no definite conclusions can be drawn from the effect of a Chlamydia trachomatis infection on the pregnancy outcome, such as birthweight and stillbirth.

In conclusion, endocervical Chlamydia trachomatis infection in pregnant women in Zaîre do occur, but further investigations of the consequences of this infection on pregnancy outcome are needed.

GERALDINE BEAUJEAN University of Limburg Hei-grindelweg 43

6414 BS Heerlen, the Netherlands INGRID WILLEMS

University of Limburg Koutenveld 6

6441 CM Brunssum, the Netherlands

We thank the Department of Medical Microbiology of the University of Maastricht, J. Philips and E. Stobberingh for their support and advice, and the staff of l'Hôpital de Kyondo.

The study was supported by a grant of de Beer Company.

1 Oriel JD, Ridgway GL. Genital infection by Chlamydia trachomatis Edward Arnold Ltd, London, 1982, p. 4-5.

2 Osoba AO. Bailliere's Clinical Tropical Medicine and Communicable Diseases Volume $2 /$ number 1 . Sexually transmitted diseases in the topics. Balliere Tindall 1987, 3-4.

3 Mabey DCW, Whittle HC. Genital and neonatal chlamydial infection in a trachoma endemic area. Lancet 1982; ii: $300-1$.

4 Marie Laga et al. Epidemiology of ophthalmia neonatorum in Kenya. Lancet 1986;ii:1145-8.

5 Bentsi C, Klufio CA, Penine PL, et al. Genital infections with Chlamydia trachomatis and Neisseria gonorrhoeae in Ghanaian women. Genitourin Med 1985;61:48-50.

6 Sweet RL, Landers DV, Walker C, Schachter J. Chlamydia trachomatis infection and pregnancy outcome. Am J Obstet Gynaecol 1987;156:824-33.

The Prevalence of Chlamydia infection in outpatient clinics in Beijing, China

SIR,-We have studied the prevalence of Chlamydia trachomatis in Beijing, China, by use of a fluorescein-labelled monoclonal antibody. ${ }^{12} \mathrm{~A}$ positive result implies the identification of at least ten elementary bodies; a negative report was based only upon adequate smears that revealed intact squamous and/or columnar epithelial cells in the specimen well.

Six hundred and seventy eight women attending the Peking Union Medical College Hospital were recruited into the study between April 1988 and May 1989. Twenty eight smears were unsatisfactory. The results of the remaining 650 smears are reported here. We found the following rates of chlamydial infection: Sexually transmitted disease clinic (27/43, $62 \cdot 8 \%$ ), Infertility clinic $(30 / 334$, $9.0 \%)$, Gynaecology clinic $(10 / 168$, $6.0 \%)$ and Obstetric clinic (3/105, $2.9 \%$ ). The highest prevalence was seen in those aged under 25 years $(9 / 33,27.3 \%)$ and in those aged 40 years and over $(7 / 29,24.1 \%)$ compared to those aged 25-39 (54/588, $9 \cdot 2 \%$ ).
The prevalence of chlamydia in 1000 women attending a gynaecology outpatient department in Hangzhou in 1986 was $1 \%{ }^{3}$ Our data demonstrate a higher prevalence in Beijing in 1989 and this may reflect an increasing incidence of chlamydial infection in China.

$$
\begin{array}{r}
\text { NI AN-PING, } \\
\text { GU CHUN-XIA, } \\
\text { LI SHI-TAI, } \\
\text { YANG HAN-YING, } \\
\text { WANG BO, } \\
\text { GAI MING-YING,\$ } \\
\text { CHEN MIN-JUN, } \\
\text { GE QIN-SHENG } \\
\text { From the Departments of Clinical } \\
\text { Laboratories, Female Infertility, } \\
\text { Gynecology, Obstetrics,\$ Dermatology } \ddagger \text { and } \\
\text { the STD clinics, Peking Union Medical } \\
\text { College Hospital, Beijing, China. }
\end{array}
$$
Address for correspondence: $\mathrm{Dr} \mathrm{Ni} \mathrm{An-}$ Ping, Department of Clinical Laboratories, Peking Union Medical College Hospital, Beijing 100730, China.

1 Milton R, Tam WE, Stamm H, et al. Culture-independent diagnosis of Chlamydia trachomatis using monoclonal antibodies. N Engl J Med 1984;310:1146-50.

2 Wiesmeier E, Bruckner D, Black $M$. Detection of Chlamydia trachomatis infection by direct immunofluorescence staining of genital secretion. Obstet Gynecol 1987;69:347-9.

3 Hodgson JE, Shi Yi-Fu, Yong-Liang Gao, Kong-Ji Wu, Bao-Yi Jiang, YuLa Chen. Chlamydial infection in a Chinese gynecologic outpatient clinic. Obstet Gynecol 1988;71:69-99.

\section{Genito-urinary medicine in rural areas in England}

Sir,-We have recently taken up posts as consultants in genito-urinary medicine (GUM) in Cumbria. Most training schemes in this speciality are based at teaching hospitals located within major cities, but some aspects of GUM practice are different in rural areas because of social or geographical factors. Training schemes could be enriched by the inclusion of a period of training in a district general hospital, and lessons learnt from rural experience may enhance the practice of GUM in city clinics.

Some rural health districts have addressed only recently the difficulties in providing a GUM service. In these districts there is a strong tradition of general practitioners attempting to treat sexually transmitted diseases, despite the disadvantages of so doing. ${ }^{1}$ 
Referral to a specialist clinic may carry a particularly heavy stigma.

Distance and travel. Cumbria is a rural area with a low population density ( 72 per $\mathrm{km}^{2}$ ); therefore patients have to travel very considerable distances in order to attend a clinic.Many patients rely on limited public transport services and travelling difficulties may be compounded by restricted clinic opening times. Public transport is also expensive and potential new patients will be unaware that they may be entitled to reclaim their transport costs. A busy city clinic enjoys the advantages of being open on a "fulltime" basis. Some clinical specimens will need to be sent to central laboratories, often located in other districts, and this introduces delays and procedural complications.

Social and sexual characteristics. Rural populations may have different priorities in life from city dwellers, and may place a low importance on those aspects of health care which they perceive as being trivial. Young people have a degree of privacy which would not be available in a city. The tourist industry attracts many young seasonal workers and provides a constant stream of new sexual partners (although many rural people do not wish to become involved with outsiders). Sexually transmitted diseases (STD) are known to be common in the tourist industry.

Confidentiality is more difficult to maintain in a small town than in a city. Any patient attending a hospital is likely to be recognised and questioned about the reason for their attendance. Some patients travel considerable distances in order to attend a clinic at which they believe they would be unrecognised.

Medical lifestyle. The rural doctor may own a substantial property, breathe clean air, live in tranquility, and be able to enjoy rural pursuits. Attending regional or national meetings may pose problems; even an audit meeting with one's nearest professional neighbour can involve a long journey. Taking annual or study leave is difficult; the consultant will not have the benefit of junior staff, and may have only occassional support from clinical assistants. Some consultant colleagues may recognise the need for a GUM service and be very supportive; others may not understand the role of this speciality. Although much time will be spent travelling by car, time spent in one's own car may be more congenial than travel by London Underground! It is difficult to undertake the care of sick inpatients when duties are split between several widely separated sites.

Management of patients. Treatment and follow-up regimes need to be kept simple to involve the minimum number of attendances, otherwise the default rate will be excessively high. Epidemiological treatment may be appropriate more frequently than in a city clinic.

In some city clinics, genital warts are treated by a different doctor or nurse at each visit, and multiple attendances are not unusual. Rural consultants who regularly review their patients will recognise the need for careful evaluation and treatment of warts at each visit.

The ideal treatment for gonorrhoea, in a patient who cannot be depended upon to reattend would be effective against both penicillin sensitive and resistant strains, would eliminate infection from all genital and extragenital sites, and would treat concurrent chlamydial infection. There is no such treatment, but it may be advantageous to treat patients with gonorrhoea for possible concurrent chlamydial infection. Purists may raise several objections; what should be written on the contact slip, what is the appropriate KC60 diagnosis, how can the overtreatment of some patients be justified? Despite these objections, concurrent anti-chlamydial treatment for patients with gonorrhoea is now considered by some authorities to be an important component of programs to control chlamydial infection. ${ }^{2}$

Repeated serological tests for syphilis could be restricted to certain patient groups, such as those with genital ulcers or with known higher risk exposures.

Contact tracing. STDs are endemic in cities but present as sporadic cases or clusters in rural areas. The value of contact tracing an index case is therefore enhanced. Indeed, effective contact tracing over a period of time could lead to a high degree of control over certain conditions. However, geographical and social factors make contact tracing difficult, and migrant workers and holiday-makers may constantly introduce new infections. Contact tracing patients infected with human immunodeficiency virus (HIV) raises many important issues. ${ }^{3}$ In rural areas with a low prevalence of HIV, the benefits of contact tracing are very clear, but the problems of maintaining confidentiality are also magnified.

DAVID COKER BELINDA STANLEY Furness General Hospital, Barrow in Furness, Cumbria, CA14 4LF

Cumberland Infirmary Carlisle, Cumbria CA2 $7 H Y$, UK

1 Coker DM, Timmins DJ, O'Mahony C. Chlamydia: one step forward or two backwards? Br Med J 1989;298:51.

2 Schachter J. Why we need a program for the control of Chlamydia trachomatis. N Engl J Med 1989;320:802-4.

3 Adler MW, Johnson AM. Contact tracing for HIV infection. $\mathrm{Br}$ Med $\mathrm{J}$ 1988;296:1420-1.

Accepted 6 February 1990.

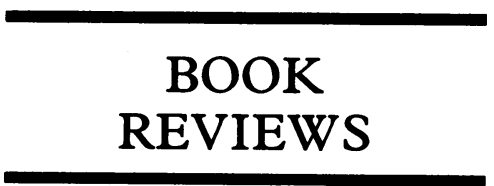

A Colour Atlas of Dermatoimmunohistocytology. By Hiroaki Ueki and Hideo Yaoita. ( $£ 60.00$.) London: Wolfe Medical, 1989.

In recent years there have been major advances in the understanding of the immunology and the immunophysiology of the skin and its function. The identification of various new antigenic structures on different types of cells and tissues, the development of monoclonal antibodies and advances in the use of various labels in detecting these cell markers has greatly helped with the diagnoses of many cutaneous disorders and has given us a better understanding of the pathogenesis of these disorders. Professors Ueki and Yaoita have produced a beautiful volume which illustrates the state of 